\title{
Instabilities in a thin current sheet and their consequences
}

\author{
N. Singh \\ Electrical and Computer Engineering Department University of Alabama, Huntsville, Al 35899, USA \\ Received: 12 March 2003 - Revised: 22 January 2004 - Accepted: 4 February 2004 - Published: 13 April 2004
}

\begin{abstract}
Using a fully 3-D particle in-cell simulation, we studied the electrodynamics of a thin current sheet (CS). Starting with a uniform plasma and anti-parallel magnetic field, Harris equilibrium is achieved during the early stage of the simulation. In the processes of reaching the equilibrium, both electrons and ions in the newly formed CS are energized and develop pitch-angle anisotropies. We find two distinct stages of primarily electrostatic instabilities; in the first stage the relative drift between electrons and ions drives the instability in the central regions of the CS. The electrostatic fluctuations scatter electrons causing current disruption in the central region. The associated reduction in the average drift velocity of the current-carrying electrons generates sheared flow. The second stage of the instability begins when the drift velocity develops a minimum in the central plane. Then the shear and the growing electrostatic fluctuations under the condition of the maintained anti-parallel driving magnetic field configuration feed each other making the instability explosive. The growing fluctuations create plasma clumps as the electrons and ions are progressively trapped in the large-amplitude waves. The density clumping also generates clumps in the current. The non-uniform current distribution causes magnetic reconnection, accompanied by heating of electrons and ion at a fast rate and nearly complete bifurcation of the current sheet. Anomalous resistivity during different stages of the evolution of the CS is calculated and compared against theory.
\end{abstract}

\section{Introduction}

One of the outstanding problems in plasma physics is the understanding of the processes, which initiate reconnection of anti-parallel magnetic fields bounding current sheets in collisionless plasmas. In recent years, several studies based on 2-D particle-in-cell (PIC) simulations have been performed to unravel the kinetic processes occurring during reconnection (Shay et al., 2001; Pritchet, 2001; Hesse et al., 2001). These studies have been very successful in revealing the variety of nonlinear processes accompanying the evolution of

Correspondence to: N. Singh

(singh@ece.uah.edu) reconnection. A bibliography for studies on magnetic reconnection is in two recent reviews by Hesse and Birn (2000) and Pritchett (2000). These 2-D kinetic studies however fail to reveal the processes, which initiate the reconnection. Over the years, it has been speculated that the reconnection might be initiated by plasma instabilities driven by the current. In order to tackle this problem 3-D kinetic simulations are needed, self-consistently including the third dimension along the current. Pritchett et al. (1996) have performed such 3-D simulations revealing the role of the drift kink instability (DKI) in the initiation process. This is an electromagnetic instability and it has been shown that it becomes ineffective for realistic electron to ion mass ratio (Daughton, 1999). Recently Zeiler et al. (2002) and Drake et al. (2003) reported studies using 3-D PIC simulations. However, even in these 3-D treatments a $X$-line was used as an initial condition to study the further evolution of reconnection, setting aside the issue of initiating reconnection by the current-driven plasma instabilities.

Lui (2000) and Kropotkin et al. (1999) have suggested that electrostatic instabilities (EI), such as the Weibel, modified two-stream and electron-acoustic modes, might initiate the reconnection process via either current disruption, generation of anomalous resistivity or nonlinear coupling between EI-driven and ion tearing wave modes. Despite these initial studies, the question about initiation of reconnection has remained largely unsettled. Saha and Singh (1999) reported preliminary results of 3-D PIC simulations showing reconnection occurring following the nonlinear evolution of ion modes (Fried and Gould, 1961).

In connection with research on solar flares (Priest, 1976) and coronal heating by nanoflares (Parker, 1972), ionacoustic and Buneman instabilities have been invoked for generating anomalous resistivity and dissipating the magnetic energy. Laboratory experiments on magnetic reconnection have also shown such instabilities at the time of the merging of magnetic field lines (Baum and Bratenaul, 1976).

The main aim of this paper is to demonstrate that electrostatic instabilities could indeed play a crucial role in dissipating magnetic energy stored in current sheets as well as in affecting magnetic reconnection. For this purpose, we simulate the processes driven in a thin current sheet, 
in which the current at early times is primarily carried by the electrons. The electron drift is sufficiently large to drive Fried-Gould ion mode instability (Fried and Gould, 1961), which is closely related to the Buneman mode (Hasegawa, 1975). This nearly purely growing instability sets up large amplitude electrostatic fields, which scatter electrons and reduce the current in the central region of the CS. The associated reduction in the electron drift generates a shear in the electron flow. The developing shear becomes a new source of free energy for the electrostatic modes, which in turn feed the shear by further reducing the current and hence the drift velocity of the current-carrying electrons in the central CS. Thus the instability becomes explosive under the condition of the maintained anti-parallel driving magnetic field configuration. The non-uniform current disruption in the explosive instability causes reconnection. The induced electric fields after the onset of reconnection cause electron and ion heating at a rate much faster than that prior to the reconnection. Furthermore, there is nearly complete bifurcation of the CS with large current densities on either side of the central CS.

The clumping of electrons and ions in the central CS during the Buneman and shear-driven instability stages serve as scattering centers and a source of anomalous resistivity as proposed by Dupree (1970) in connection with ion-acoustic turbulence. However, we find that the purely growing sheardriven waves overwhelm any effect of anomalous resistivity driven tearing of the current sheet. The waves are by themselves effective in clumping and tearing of the CS.

It is worthwhile to point out that there is a vast literature on instabilities in thin current sheets. Recently Silin and Büchner (2003a,b) studied the kinetic instabilities of thin current sheets showing that the drift-resonant instability occurs when the lower-hybrid-drift waves (Ozaki et al., 1996) penetrating from the outer parts of a current sheet into its central part resonantly interact with the drifting ions. They have compared and contrasted their findings with the findings of several prior studies on the topic. All theses prior studies are based on an initialized Harris equilibrium, in which ions are the major current carriers. In contrast, we deal here with a current sheet, which is continually driven by external antiparallel magnetic fields simulating a continued forcing as in situations of nanoflares (Parker, 1972), solar flares (Priest, 1976) as well as generalized magnetic turbulence( $\mathrm{Wu}$ and Chang, 2000; Matthaeus et al., 2003). The induced electric field in the forced situation preferentially accelerates the unmagnetized electrons in the central current sheet and they become the major current carriers as found in laboratory experiments (Baum and Bratenaul, 1976). We have attempted to simulate such a situation and found that during the initial stages of the evolution of the current sheet the electrostatic ion mode instabilities dominate and not the electromagnetic instabilities as found in several prior studies with the initialized Harris equilibrium.

The rest of the paper is organized as follows. In Sect. 2 we describe the method of simulation. Section 3 deals with setting up of the CS including its structure, acceleration of current carrying electrons and ions and their velocity distri- bution functions. Section 4 deals with ion-mode instabilities driven by the electron drift followed by formation of shear and shear driven explosive waves. Section 5 deals with the consequences of the explosive instability. The issue of the generation of anomalous resistivity is discussed in Sect. 6. Conclusions of the paper and their relevance to space and astrophysical plasmas are discussed in Sect. 7.

\section{Method of simulation}

We utilize a fully 3-D electromagnetic PIC code with relativistic effects included (Birdsall and Langdon, 1985). We used this code to simulate a volume of plasma given by $L_{x} \times L_{y} \times L_{z}=32 \times 32 \times 64 \lambda_{d}^{3}$, where $\lambda_{d}^{3}$ is the Debye length. The anti-parallel magnetic field is given by

$\boldsymbol{B}=B_{o} \tanh \left(\left(z-z_{c}\right) / D\right) \boldsymbol{a}_{x}$,

where $B_{o}$ is the magnetic field at distances far away from the central plane $z=z_{c}=L_{z} / 2, D$ is the scale length in the variation of $B_{x}$ with $z$. This field in Eq. (1) is always maintained through the entire simulation time as an external source for driving the plasma. The plasma is initially uniform and it has no current in it. For the driving field in Eq. (1) starting at time $t=0$, the simulation self-consistently yields the Harris equilibrium (Harris, 1962). We point out here that it is not essential to initialize the simulation with Harris type of density distributions at time $t=0$, instead the equilibrium is reached as the plasma and the fields evolve self-consistently. In the simulation the grid spacing is the Debye length, $\lambda_{d o}$, in the initial plasma with a uniform density $n_{o}$ and Maxwellian electrons and ions having temperatures of $T_{e o}$ and $T_{i o}$, respectively. The ion to electron temperature ratio is 2 . We incorporated periodic boundary conditions along $x$ and $y$ on both particles and fields. Along $z$ we used a conducting boundary conditions for the fields while the particle boundary conditions were varied in different simulations. In the simulations described here the particles leaving the simulation box at boundaries $z=0$ and $L_{z}$ are not recirculated, instead they are replaced by new ones from the Maxwellian distributions having the same temperatures as the initial plasma loaded in the simulation box. The fields in this code are updated solving the time-dependent Maxwell's equations (Birdsall and Langdon, 1985), including a correction for the space-charge field (Langdon, 1992).

We performed simulations for two values of the ratio of velocity of light, $C$, to the electron thermal velocity, $V_{t e o}, C / V_{t e o}=10$ and 25 and found physically similar results. The ion to electron mass ratio was $M / m=16$. We chose $\boldsymbol{B}_{\boldsymbol{o}}$ so that electron cyclotron frequency $\Omega_{c e}=0.5 \omega_{p e o}$, where $\Omega_{c e}$ and $\omega_{\text {peo }}$ are the electron cyclotron frequency and the plasma frequency with the initial density $n_{o}$. The electron and ion skin depths are $l_{e}=c / \omega_{\text {peo }}=10 \lambda_{d o}$ and $l_{i}=c / \omega_{\text {pio }}=40 \lambda_{d o}$ and the corresponding Larmor radii are $\rho_{e}=2 \lambda_{d}$ and $\rho_{i}=12 \lambda_{d}$. We choose $D=12 \lambda_{d o} \sim \rho_{i}<l_{i}$, but $D \sim l_{e} \gg \rho_{e}$. In the discussions below unless mentioned otherwise, the units are 

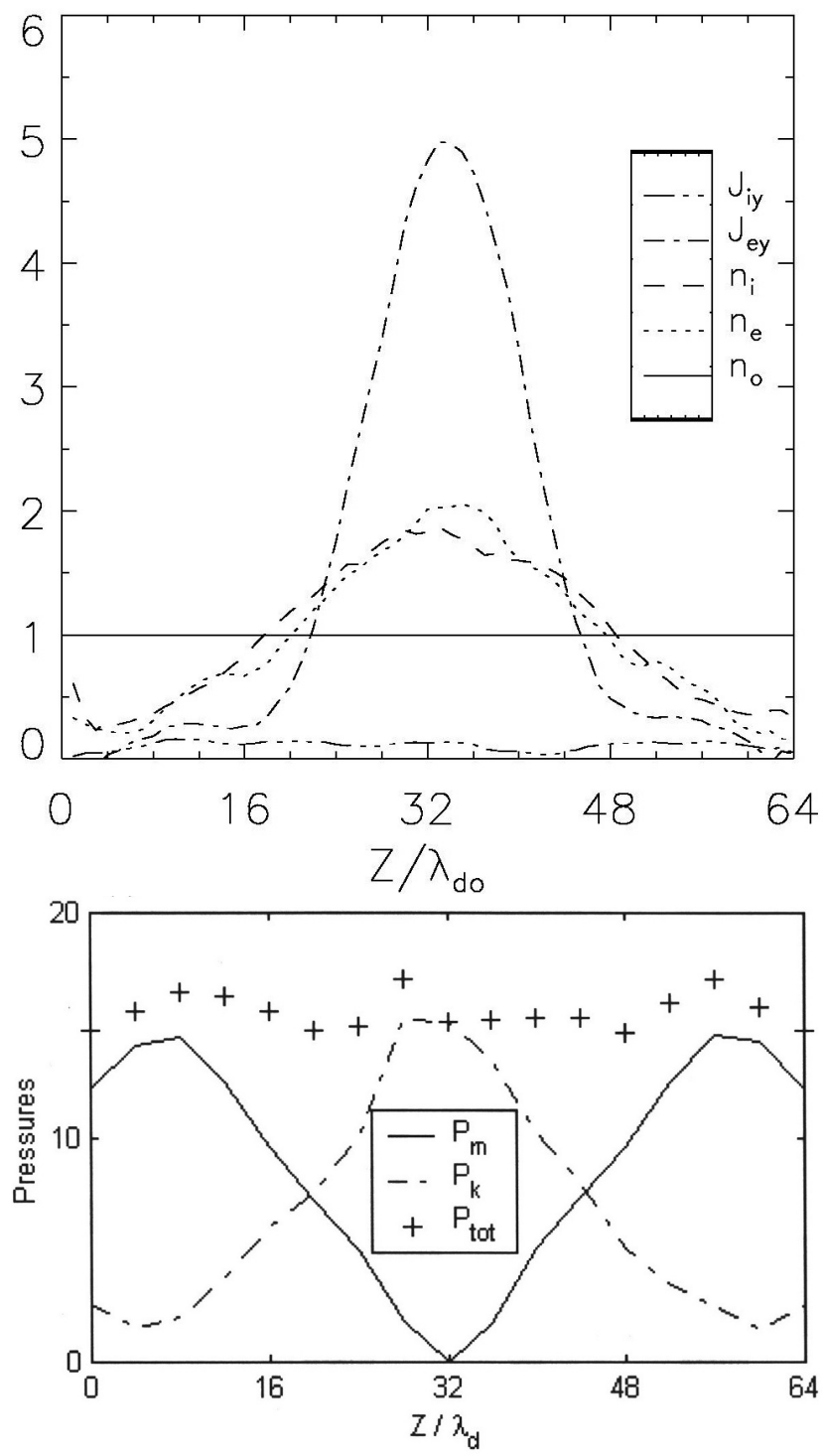

Fig. 1. Current sheet at an early time $t=50$. (a) Distributions of $n_{e}(z), n_{i}(z), J_{e y}(z)$ and $J_{i y}$ across the current sheet. (b) Pressure balance at $t \sim 50$; distributions of magnetic $\left(P_{m}\right)$, kinetic $\left(P_{k}\right)$ and total $\left(P_{t}\right)$ pressures.

$\omega_{\text {peo }}^{-1}, \lambda_{d o}, V_{\text {teo }}, K_{b} T_{o} / e \lambda_{d}, m \omega_{\text {peo }} / e$, and $n_{o} e V_{\text {teo }}$ for time, distance, velocity, electric field, magnetic field and current, respectively. We started the simulations with 36 pairs of electrons and ions in each cell of size $\lambda_{d}^{3}$. At any time there were $\sim 2.5 \times 10^{6}$ electrons and ions in the simulation box. Since we use open boundary conditions for the particles at $z=0$ and $z=L_{z}$, this number varies a little bit $(\sim 5 \%)$ over the course of the run. The time step was $\delta t=0.02 \omega_{\text {peo }}^{-1}$, which insured stability of the simulations through out the run.

\section{Setting up the current sheet and its stability}

As mentioned above we start the simulation without a current sheet but with a reverse magnetic field configuration applied

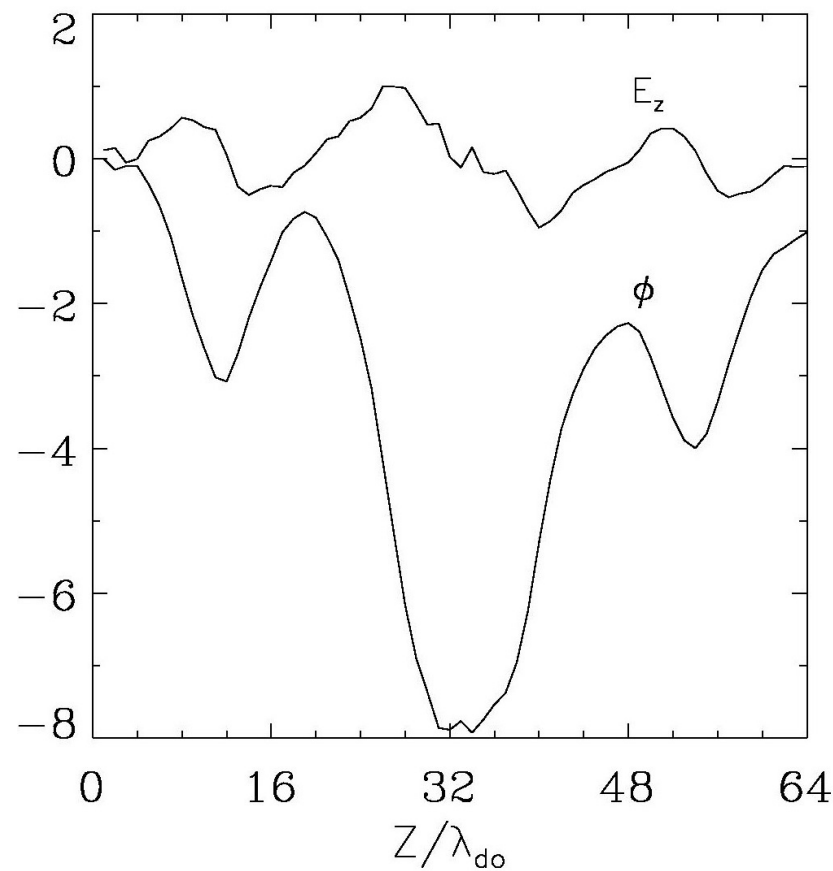

Fig. 2. Electric field $E_{z}(z)$ and potential $\varphi(z)$ at $t=50$ during the formation of the CS.

to quasi-neutral uniform plasma. As the simulation runs, quite soon a current sheet is self-consistently set up in the plasma. Figures $1 \mathrm{a}$ and $1 \mathrm{~b}$ show the state of the current sheet at time $t=50$. The normalized electron $\left(n_{e}\right)$ and ion $\left(n_{i}\right)$ densities and the corresponding current densities $J_{e y}$ and $J_{i y}$ at $t=50$ are shown in Fig. 1a; the particle and current densities peak in the central plane $z=32$. The ion current is quite small compared to the electron current. Thus the total current is $J_{y} \cong J_{e y}$. The particle density maximums are approximately $\sim 2 n_{o}$ while the current maximum is $J_{y m} \sim 5$. Using the Ampere's law, the maximum current is given by $J_{y m}=B_{o} / \mu_{o} l \approx(50 / D)\left(n_{e} e V t h\right)=4.2\left(n_{o} e V t h\right)$, which matches well with the above result from the simulation (Fig. 1a). Figure 1b shows the distribution of magnetic, kinetic and total pressure along $z$ at time $t=50$. It reveals that the state of plasma is nearly in equilibrium as the total pressure, which is the sum of the magnetic and kinetic pressures, is nearly constant.

As mentioned above initially $J_{y}=0$, but in a short time the current builds up. We describe here the plasma response during the course of current build up. According to the Maxwell equation magnetic flux given by Eq. (1) generates positive electric field $E_{y}$. This field accelerates the plasma electrons, which carry the current. The current carrying electrons feel a $\boldsymbol{V} \times \boldsymbol{B}$ force towards the central parts of the current sheet, transporting them toward the plane $z \sim 0$. Likewise ions are also transported as they, being un-magnetized, feel a force via electrostatic coupling with the electrons. Thus the initial uniform density is modified, peaking near the central plane (Fig. 1a). Figure 2 shows the electrostatic field $E_{z}$ resulting from this process. Even though the field is oscillatory, 

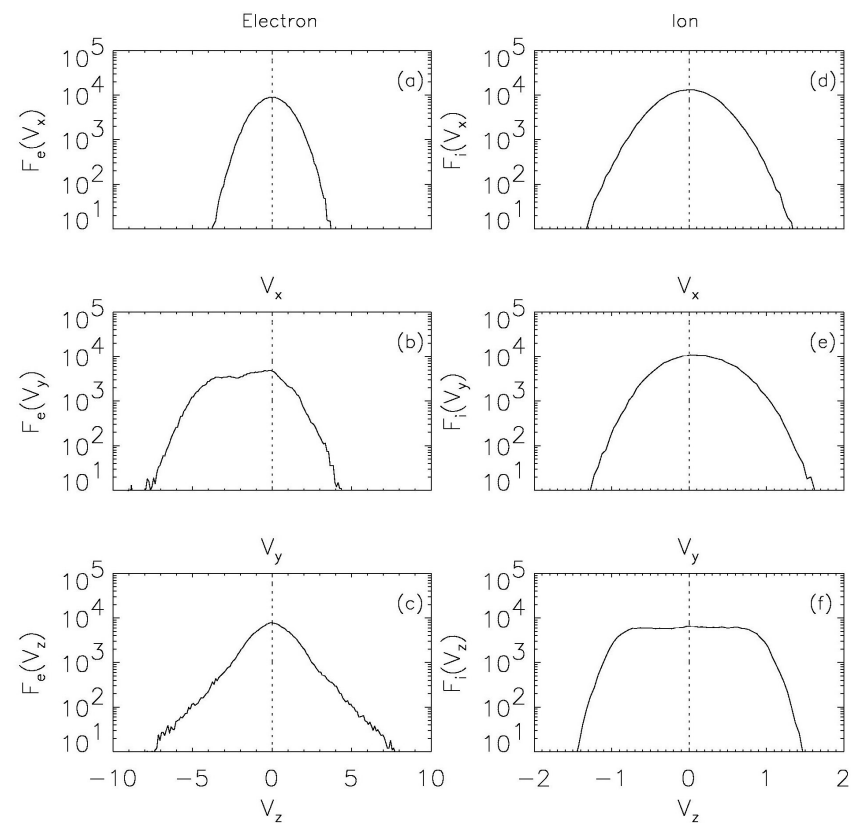

Fig. 3. Electron (left panels) and ion (right panels) velocity distribution functions at $t=50$. Note the electrons, which carry the current, have a velocity distribution $F_{e}\left(V_{y}\right)$ shifted toward the large negative velocities. Also note the flat-top distribution $F_{i}\left(V_{z}\right)$ for the ions.

on the average it points toward the $z=0$ plane. The corresponding potential distribution $\varphi(z)$ is also plotted in Fig. 2, showing a deep potential well for the ions centered at $z=32$. The evolving fields modify the electron and ion velocity distribution functions. Figure 3 shows the velocity distributions of electrons and ions in the CS $(20<z<44)$ at $t=50$. The left-hand panels show the electron distribution functions $F_{e}\left(V_{x}\right), \quad F_{e}\left(V_{y}\right)$ and $F_{e}\left(V_{z}\right)$ from top to bottom, respectively. The corresponding ion distributions $F_{i}\left(V_{x}\right), F_{i}\left(V_{y}\right)$ and $F_{i}\left(V_{z}\right)$ are shown in the right-hand panels. The $V_{x^{-}}$ distributions for the electrons and ions are nearly the same as the initial ones. The $V_{y}$ - and $V_{z}$-distributions for the electrons are significantly modified; $F_{e}\left(V_{y}\right)$ has a net drift in the negative $y$ direction that supports the current $J_{y}$ and it also appears to be heated compared to $F_{e}\left(V_{x}\right) . F\left(V_{z}\right)$ for the electrons shows two populations; a cold one near $V_{z} \sim 0$ like $F\left(V_{x}\right)$ and a warm one. The cold population is the initial Maxwellian and the warm one is the consequence of acceleration in the $-y$ direction followed by their cyclotron motion. As expected, the $V_{y}$-distribution for the ions, $F_{i}\left(V_{y}\right)$, has a small net drift in the positive $y$ direction making a small contribution to $J_{y}$ from the ions. The $V_{z}$-distribution, $\left(F_{i}\left(V_{z}\right)\right)$, of ions is significantly modified because of the trapping of ions in the potential well shown in Fig. 2. The width of the nearly flat-top distribution in Fig. $3 \mathrm{f}$ is determined by the amplitude of the potential well shown in Fig. 2. An important fact to note from the discussion here is that both ions and electrons have developed thermal anisotropies with higher effective temperatures across the magnetic field than that parallel to it.

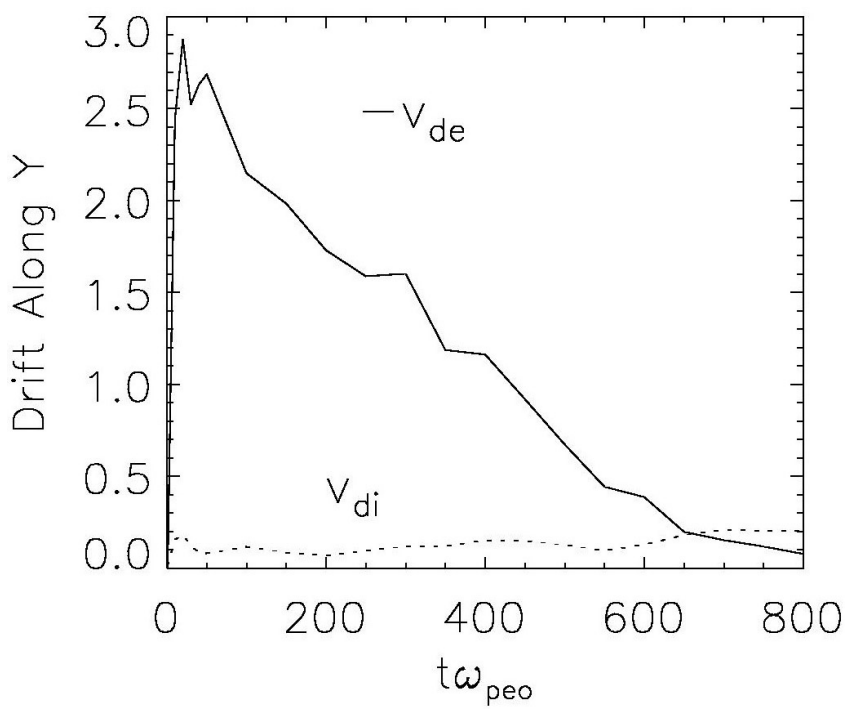

Fig. 4. Averaged drifts of (a) electrons and (b) ions lying in the central CS $(31<z<33)$.

The electron and ion $V_{y}$-distributions in Fig. 3 show that there is a relative drift between electrons and ions in the $y$ direction. Figure 4 shows the average electron $\left(V_{d e}\right)$ and ion $\left(V_{d i}\right)$ drifts along the $y$ direction as functions of time. These drifts are obtained by averaging the $V_{y}$ component of the velocities of electrons and ions in a layer of thickness $\Delta z=2$ extending in the entire $x-y$ plane centered at $z=32$. It is seen that $V_{d i}$ remains small and it slightly increases over the time span of the plot. On the other hand, $V_{d e}$ steadily decreases after $t \sim 50$. We find that until about $t \sim 300$ the relative drift $V_{r}=V_{d i}-V_{d e}$ remains large enough to drive two-stream instability for the prevailing electron and ion temperatures in the plasma. In the process of reaching the early equilibrium in the CS, the electron temperature $T_{e y}$ is enhanced to about $2.5 T_{o}$ and the electron thermal velocity to $1.6 V_{\text {teo }}$. Figures $5 \mathrm{a}$ and $\mathrm{b}$ show the examples of wave pattern generated by the instability at an early time $t=200$; they show the patterns of $E_{y}$ in the $x-z$ plane at $y=16$ and in the $x-y$ plane at $z=32$, respectively. Note the periodic wave pattern in the $x-y$ plane (Fig. 5b) is mainly confined within the current sheet (Fig. 5a). The same wave pattern is seen in Figs. $5 \mathrm{c}$ and $\mathrm{d}$ for $t=350$, except for larger amplitudes as shown by the scales on the right. Fourier Transforms of the 2-D data plotted in Fig. 5b and d show that the dominant wave mode is $(6,3)$, implying that the wavelength along $x$ is $\lambda_{x}=L_{x} / 6=5 \lambda_{d}$ and $\lambda_{y}=L_{y} / 3=11 \lambda_{d}$ and the correspondingly wave number components $k_{x}=1.3 \lambda_{d}^{-1}$ and $k_{y}=0.55 \lambda_{d}^{-1}$. These wavelengths can be also directly determined by the periodicities along $x$ and $y$ directions in Figs. $5 \mathrm{~b}$ and $\mathrm{d}$. The wave pattern inclination from the $y$ direction is at an angle $\theta_{y}=\tan ^{-1}\left(k_{x} / k_{y}\right) \approx 66^{\circ}$. Thus the ion modes are at an oblique angle to the relative drift along $y$. Since the CS is bounded and non-uniform in $z$, the linear instability analysis for one-dimensional propagation is 

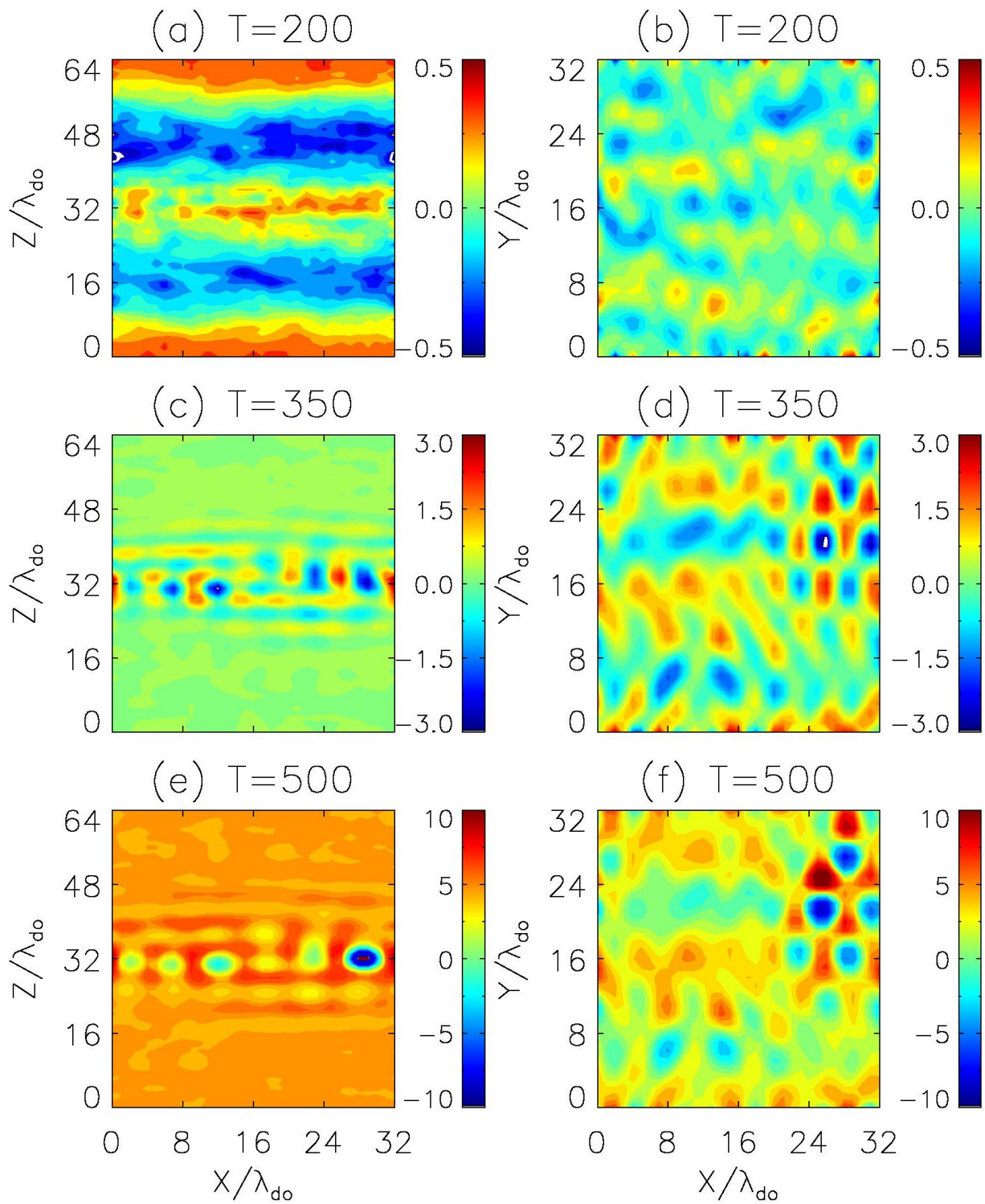

Fig. 5. Wave structures in $E_{y}(x, y=16, z)$ in the left column and $E_{y}(x, y, z=32)$ in the right column. (a)-(b) $t=200$, (c)-(d) $t=350$, and (e)-(f) $t=500$. Note that the wave structure is periodic in the $x-y$ plane and it is confined inside the current CS.

not directly applicable to the numerical calculation here. We repeated the simulation by reducing the magnetic field from $B_{o}=0.5$ to $B_{o}=0.2$ in Eq. (1) and thus reducing the current density $J_{y}$ and $V_{d e}$ by a factor of $\sim 2.5$. We found that the current sheet remained stable over the time interval discussed in this paper. It is important to note that the CS for $B_{o}=0.2$ becomes stable because $V_{d e}$ falls below the critical drift for the instability of ion modes found here.

\section{Development of shear and explosive instability phase}

The electric field fluctuations shown in Figs. 5a-d scatter electrons and begin to reduce the electron drift in the central region of the sheath. Figure 6 shows the evolution of the profile of $\left(-V_{d e}(z)\right)$. The profiles shown in the figure are obtained by dividing the plasma along $z$ in 32 layers, each of thickness $\Delta z=2$ and extending over the entire $x-y$ plane. $V_{d e}(z)$ is obtained by averaging velocity $V_{y}$ of the electrons in each layer. The numbers on the curves indicate the time when multiplied by 100 . The drift velocity steadily decreases in the central CS $(z \sim 32)$ as also inferred from Fig. 4 and it increases in the off-central regions. As mentioned above the decrease is a consequence of the scattering of the electrons by the growing fluctuations in the central CS. The decrease in $\left(-V_{d e}(z \sim 32)\right)$ causes current disruption and therefore the slope $d B_{x}(z) / d z$ near $z \sim 32$ decreases. Since the imposed magnetic field is maintained, opposing magnetic fields develop on both sides of the null plane $(z=32)$ and partly cancel the imposed field. The resultant magnetic field develops larger slope in the off-central region, where the drift velocity and the current density develop new peaks. Figure 6 


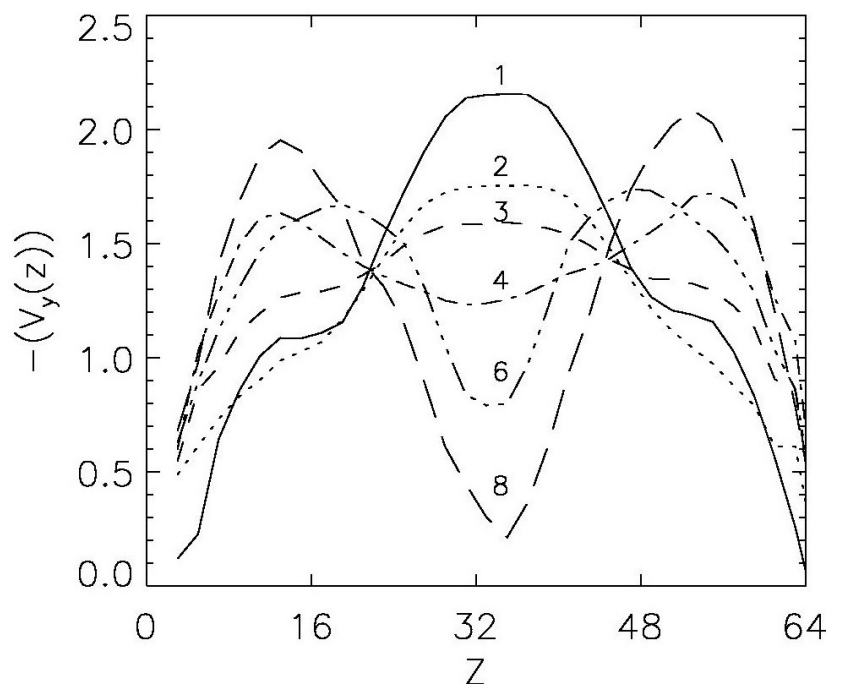

Fig. 6. Evolution of the profile of the electron drift velocity $\left(-V_{d e}(z)\right)$. Note the sheared flow with inflection points in the CS after $t \sim 400$.

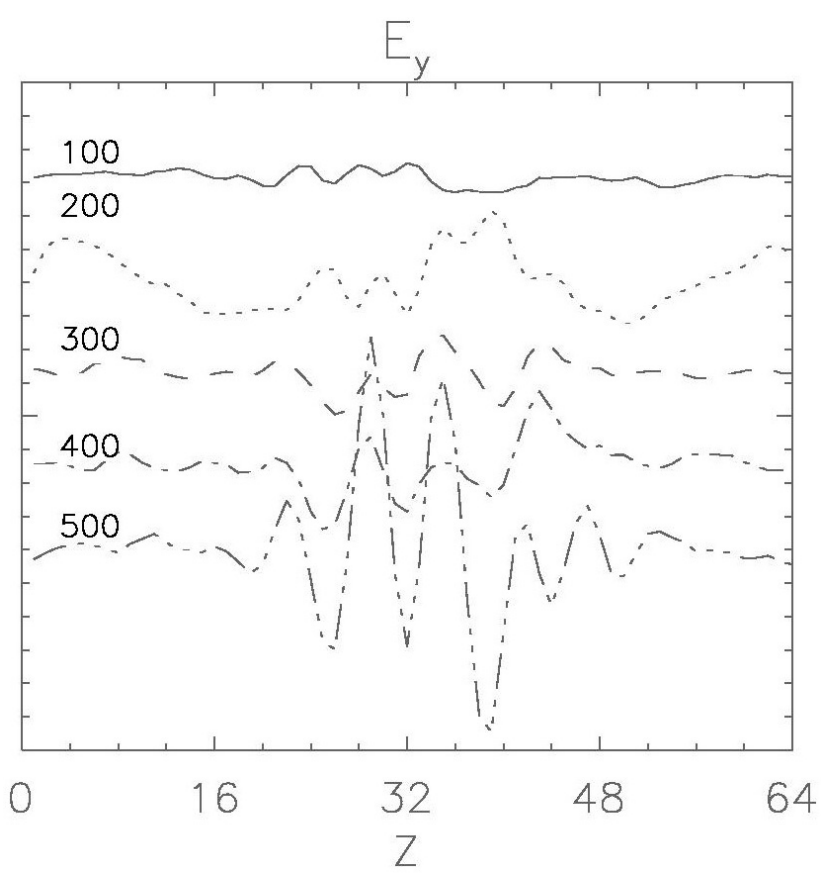

Fig. 7. Mode structure in the CS. The curves for $t<400$ show the mode structure driven by the ion mode instability. Note the large growth in $E_{y}$ for $t>400$ occurs when the current-carrying electrons develop the sheared flow shown in Fig. 6.

shows that for $t \geq 400$ the drift distribution has a minimum at $z \sim 32$ and maxima in the off-central regions. Such a configuration of $V_{d e}(z)$, having shear and inflection points, is unstable. Ganguli et al. (1988) and Romero et al. (1992) have shown that the free energy for wave excitation is proportional to the second derivative of $V_{d e}(z)$. The inflection points ensure that the second derivative changes sign in the sheared flow as required for the instability according to Raleigh theo-

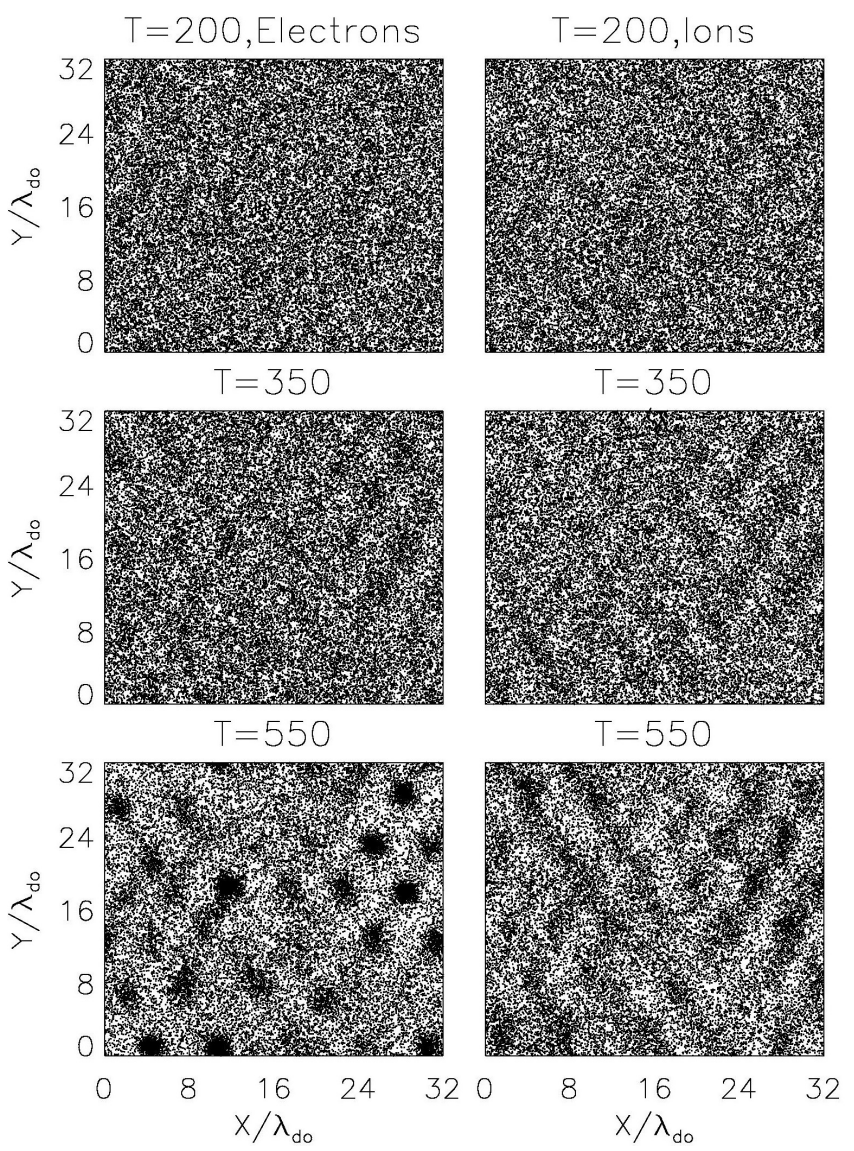

Fig. 8. Spatial distribution of electrons and ion in the $x-y$ plane. Note the formation of clumps.

rem (Mikhailovskii, 1974). Figures 5e and $\mathrm{f}$ show the further growth in the wave structure at $t=500$. Figure 7 shows the growing wave mode in the CS; the oscillations in $E_{y}$ in the CS seen for $t<400$ basically represent the wave structure appearing in Figs. 5a-d and they are driven by the two-stream instability. The curve for $t=500$ shows the beginning of the fast growing waves fed by the sheared flow in the central CS. The growing electrostatic perturbations in $E_{y}$ further scatter electrons and reduce the drift velocity in the central CS. This further enhances the shear and growth of the waves, making the instability explosive.

\section{Consequences of the explosive instability}

We describe here the plasma processes occurring in the CS after the onset of the shear formation including its fragmentation, magnetic reconnection and fast heating of both electrons and ions.

\subsection{Fragmentation of plasma and the CS}

A major consequence of the explosive phase of the instability is that clumps grow in both electron and ion densities. Figure 8 shows the evolution in the formation of clumps in 

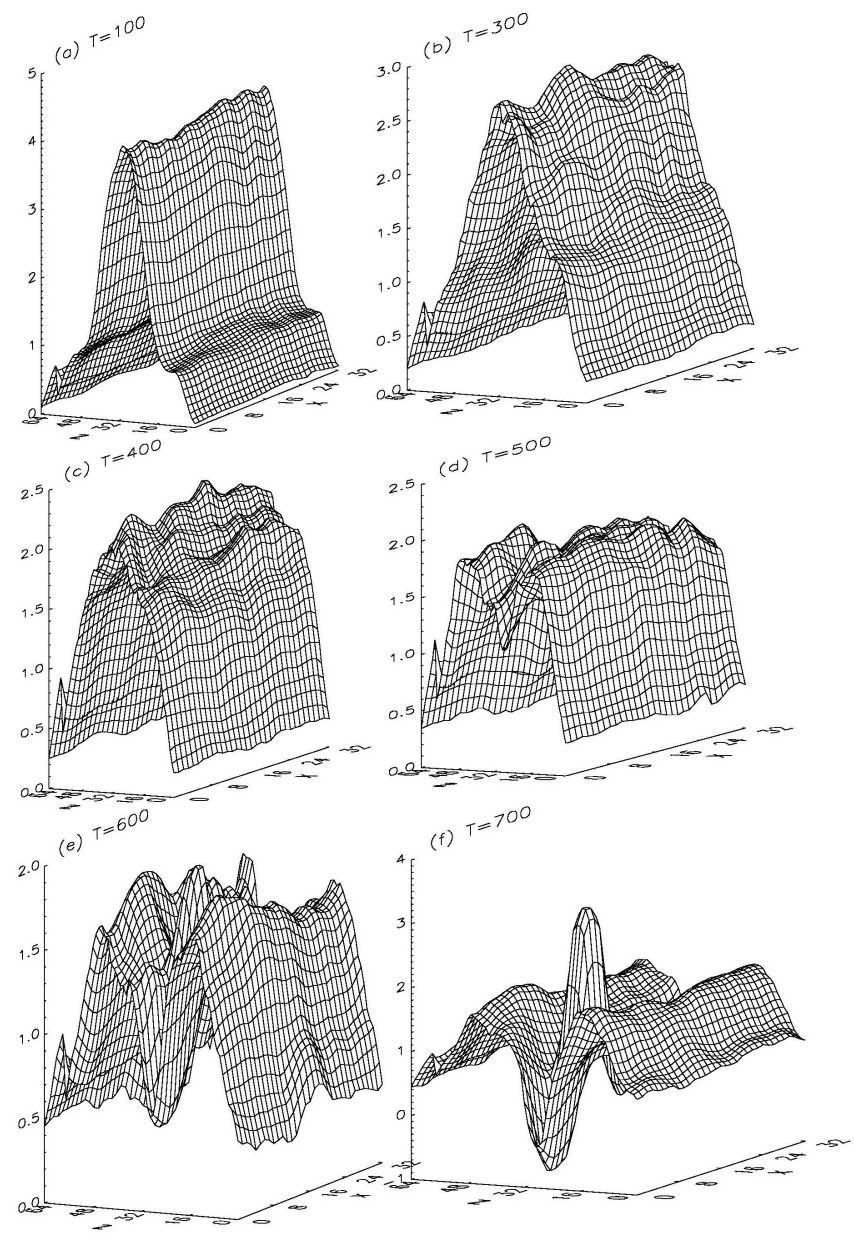

Fig. 9. Surface plots of current distribution in the $x-z$ plane. Note that the initial CS first broadens and then bifurcates as the current disrupts in the central region.

the spatial distributions of electron and ions in the $x-y$ planes occupying a volume in the central CS $(24<z<40)$. The dots in Fig. 8 represent electrons and ions and give their locations. At early times, like at $t=200$, electrons and ions are nearly uniformly distributed. The clumps are beginning to appear at $t=350$ and they are fully formed by the time $t=550$. The main cause of the clump formation is the trapping of electrons in the potential wells set by the instability. Ions also clump as they are coupled to the electrons by electric fields.

The clumping of the plasma generates clumps in the current distribution. Figure 9 shows the evolution in the current structure. The panels in Fig. 9 show the surface plots of the electron current in the $x-y$ plane at $y=9$. Physically similar current distributions appear at any $y$. At $t=100$ we see a relatively narrow current distribution as required by the imposed magnetic field. As time increases the current sheet develops wavy structures, current level drops and it also broadens. This behavior continues until $t \sim 400$. Beginning at about $t \sim 500$ the perturbations in the current in the central CS grow to quite large amplitudes and the CS begins to bifurcate with current disruptions extending along $x$. The bi-
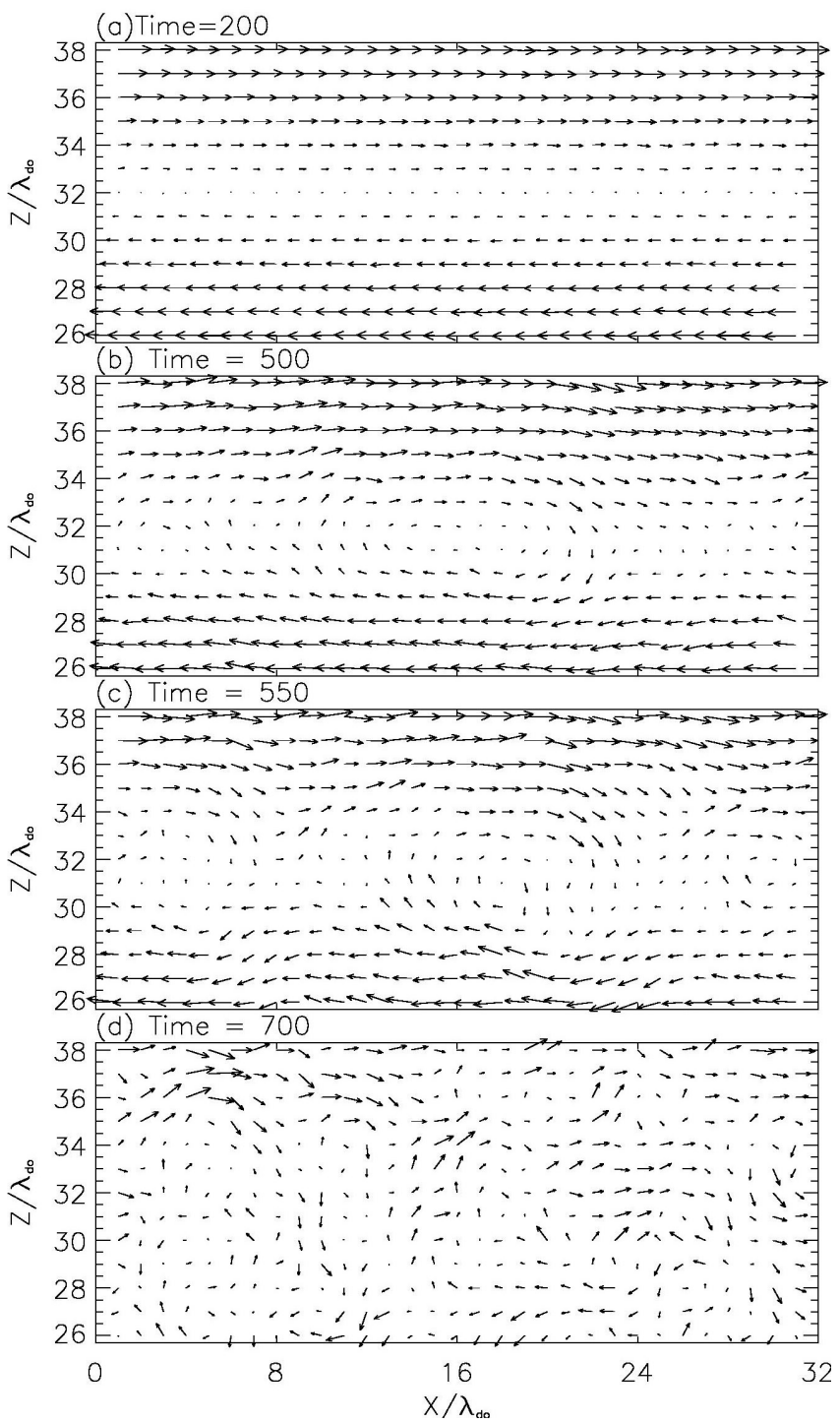

Fig. 10. MFC resulting from magnetic reconnection: vector plots at (a) $t=200$, (b) $t=500$, (c) $t=550$ and (d) $t=700$. The arrows show the direction and their lengths show the relative magnitude of the magnetic field.

furcation is clearly evident from the surface plots for $t>500$, except for some patches of large currents. As discussed later, these patches are associated with magnetic turbulence.

\subsection{Magnetic reconnection}

A significant consequence of the evolution involving electron and ion clumping, non-uniform current disruption is the merging of the anti-parallel magnetic field lines. Figure 10a shows the magnetic field vectors at $t=200$ in the $x-y$ plane at $y=17$; the magnetic field vectors are still anti-parallel with the $z$-component of the field $B_{z} \sim 0$. This initial feature of the magnetic field configuration (MFC), consisting of only the anti-parallel magnetic fields, continues until about $t \sim 400$. The vector plot at $t=500$ in Fig. 10b shows that a large magnetic loop has formed centered at $x \sim 16$. The MFC 


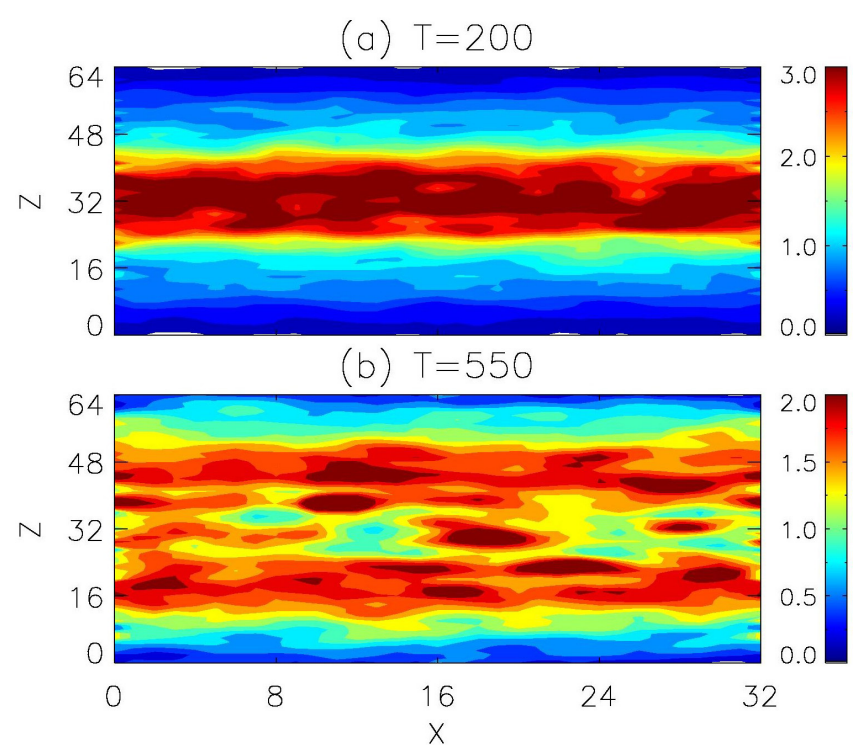

Fig. 11. $J_{y}(x, y=17, z)$ associated with the MFC in (a) Fig. 10a and (b) Fig. 10c. The patches of large currents in the CS at $t=550$ are collocated with the magnetic loops in Fig. 10c.
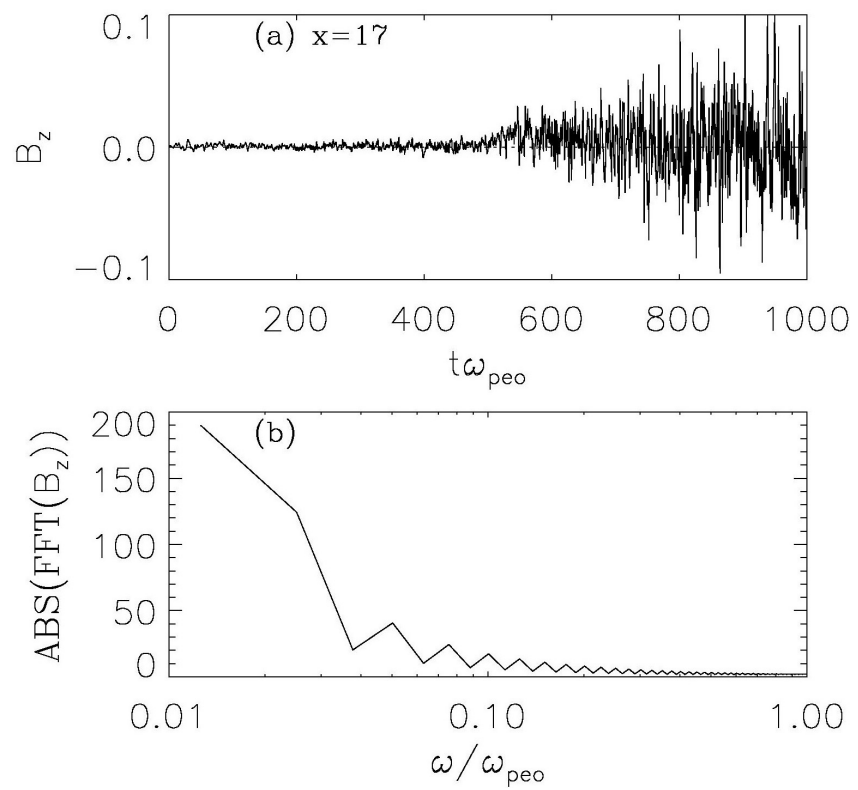

Fig. 12. (a) Temporal evolution of $B_{z}$ at $(16,17,32)$ and (b) its Fourier spectrum. The oscillations in $B_{z}$ are at low frequencies, namely, $\omega / \omega_{p e} \leq 0.03$, which is the ion cyclotron frequency with the magnetic field $B_{o}$.

changes with the current. Figure $10 \mathrm{c}$ shows that at $t=550$ MFC consists of two regions of connected fields with a loop in between. Figures 11a and b show the current structures associated with the MFC in Figs. 10a and c, respectively. Figure 11a shows that the original CS is nearly intact at $t=200$. The fragmentation of the CS is seen at $t=550$ in Fig. 11b; the loops in the magnetic field in Fig. 10c are collocated with peaks in the current distribution near the plane $z \sim 32$. The
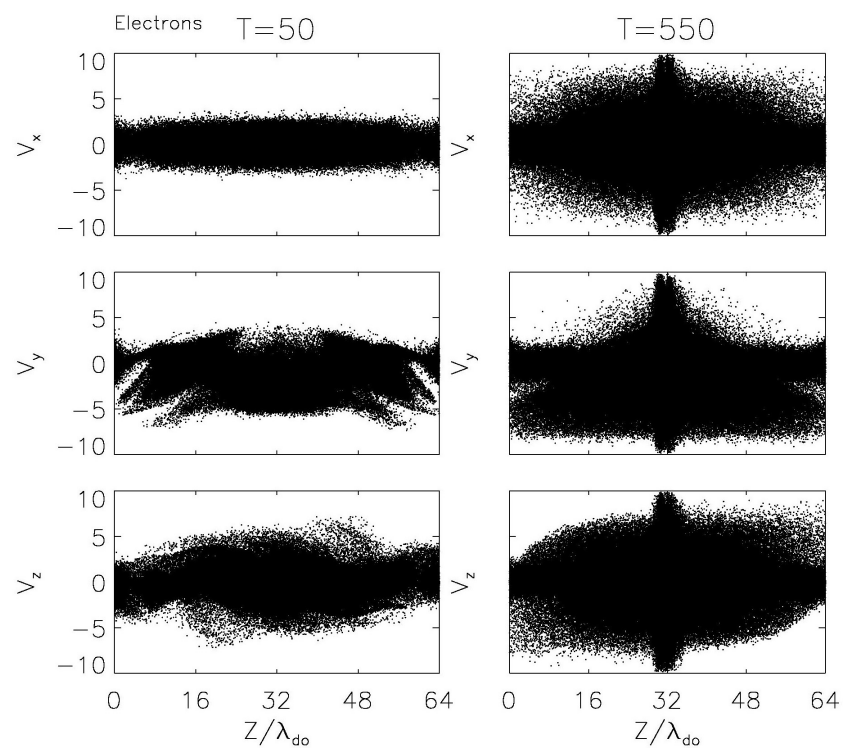

Fig. 13. Comparison of electron phase space in $z-V_{x}$ (top), $z-V_{y}$ (middle) and $z-V_{z}$ (bottom) planes at $t=50$ (left panels) and $t=550$ (right panels). At the latter time electrons have undergone large accelerations; the largest accelerations occur in the central part of the current sheet.

temporal variability of $B_{z}$ seen in the MFC after the onset of the magnetic reconnection is shown in Fig. 12a by plotting $B_{z}(16,16,32)$. The frequency spectrum of the data for $500<t<1000$ is plotted in Fig. $12 \mathrm{~b}$, which shows that the oscillations are at low frequencies below the maximum ion cyclotron frequency $(\sim 0.03)$ corresponding to $B_{o}=0.5$.

\subsection{Electron and ion acceleration}

Figure 13 shows the phase space plots of the electron in $z-V_{x}, z-V_{y}$ and $z-V_{z}$ planes at $t=50$ (left-hand panels) and $t=550$ (right-hand panels). Corresponding plots for ions are in Fig. 14. Comparing left- and right-hand panels in Fig. 13, we find that electrons are significantly accelerated in all the three velocity components at $t=550$ compared to their velocities at $t=50$. Some structural features in the phase space planes are also noteworthy. At $t=50$, the cold thermal electrons have nearly retained their initial distribution in the $z-V_{x}$ plane. The distribution in $V_{y}$ at $t=50$ has developed some complex features associated with the current flow in the positive $y$ direction; in the central region near $z=z_{c}$ electrons are heated as well as accelerated in $-y$ direction. The $z-V_{z}$ distribution at $t=50$ shows a warm electron population in the central region near $z=z_{c}$. We also see from the $z-V_{z}$ plot that electrons are flowing into the central CS from the regions $z<16$ and $z>48$. These features of the early evolution were discussed earlier in connection with the formation of the current CS (Figs. 1-3). The electron phase space at $t=550$ show that the most energetic electrons are near the center of the initial CS, namely, near $z=32$ and they appear to be nearly isotropic. Electrons accelerated in the central CS become relativistic as their velocities approach $C$. 

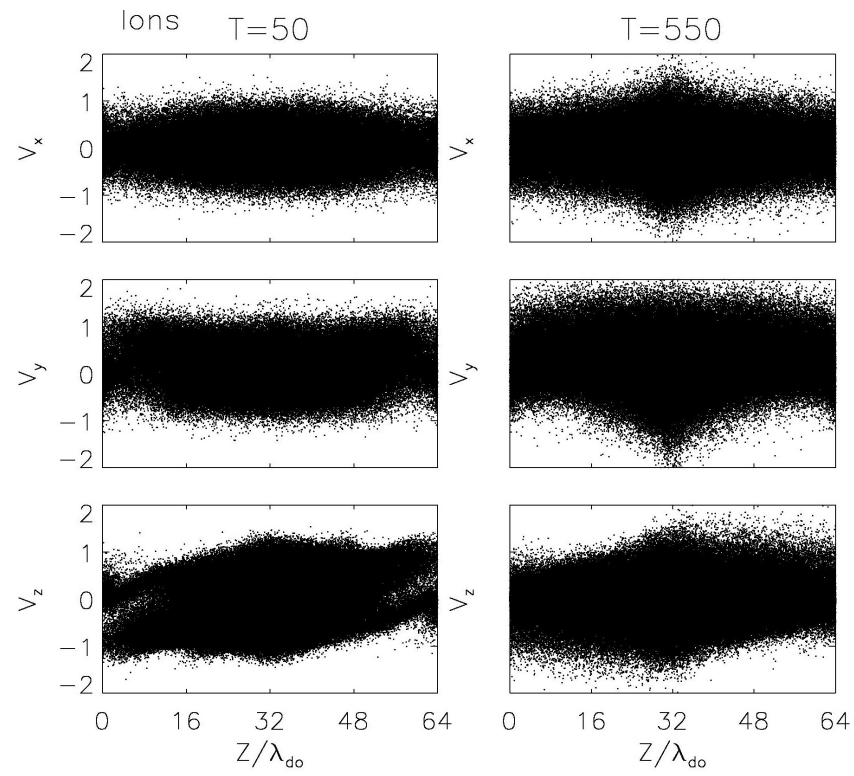

Fig. 14. Same as Fig. 13, but for ions.

The symmetry of the $z-V_{y}$ plot with respect to $V_{y}=0$ near $z \sim 32$ at $t=550$ implies that the current in the central region is disrupted as discussed earlier in connection with Fig. 9. In contrast to this, the asymmetry persists in the regions out side the central CS; in the outer region the current carrying electrons have net drifts in the negative $V_{y}$ direction. The cold electron populations appearing near $z=0$ and $z=64$ are the product of boundary condition; the electrons leaving the simulation box at $z=0$ and $z=64$ are replaced by the initial cold population.

The $z-V_{z}$ plot at $t=550$ shows that the accelerated electrons are flowing out of the central regions as revealed by the populations for $V_{z}>0$ over $48<z<64$ and for $V_{z}<0$ over $0<z<16$. Such an outflow along with $x$-directed outflows is a characteristic feature of magnetic reconnection. In our simulations the $x$-directed outflow is masked by the use of periodic boundary conditions on the particles at $x=0$ and $x=L_{x}$.

The ion phase space at $t=50$ (right-hand panels, Fig. 14) do not show any noteworthy feature, except that ions are heated in the central region in $V_{z}$ as indicated by $F_{i}\left(V_{z}\right)$ in Fig. 3. All the three left-hand panels in Fig. 14 show that after the onset of reconnection ions have also undergone a considerable heating. The heating is again largest in the central CS. Like the electrons, $z-V_{z}$ plot for the ions also show the outflow.

Figure 15 shows the time history of kinetic energy gain by electrons and ions. In this figure we have plotted average energy of the electrons lying in a slice of plasma of thickness $\Delta z=2$, centered at $z=32$, extending over the entire $x-y$ plane of the simulation. Since electrons reach relativistic velocities, the kinetic energy is calculated by $W=\left(m-m_{o}\right) x C^{2}$, where $m_{o}$ is the rest mass and $m$ the mass at a velocity $V$. We find three stages of energy gain by

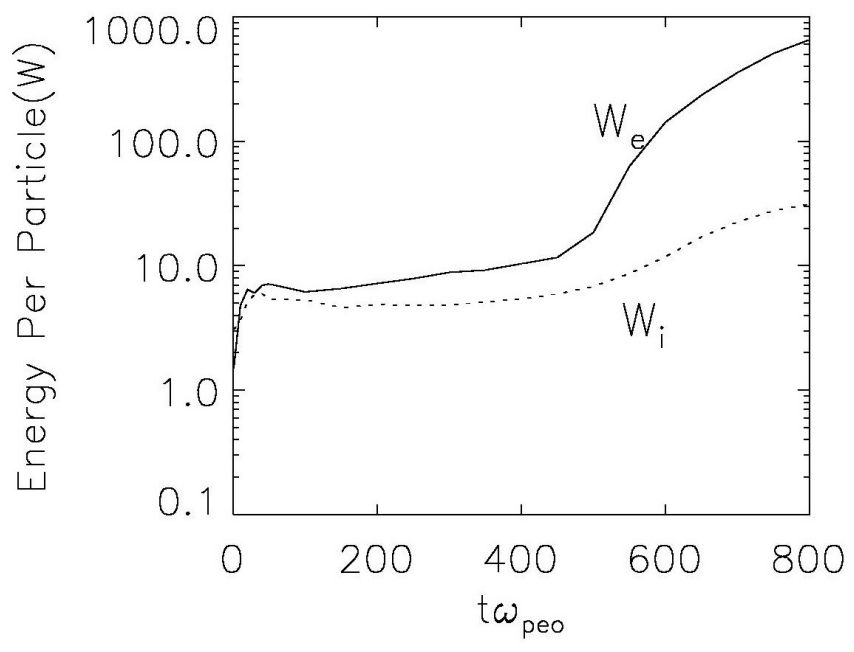

Fig. 15. Temporal behavior of the gain in kinetic energy of electrons and ions.

the electrons and ions. The first stage occurs with the CS formation; The application of the magnetic field to the plasma generates electric field $E_{y}$, which accelerates electrons near $z \sim 32$ in the $y$ direction. Some of these electrons also show acceleration in the $z$ direction as a consequence of their cyclotron motion in the anti-parallel magnetic field. These features are clearly shown by the distribution functions in Fig. 3 and the phase space plots in Fig. 13. In this phase the unmagnetized ions gain energy in the $V_{z}$ component as seen in Figs. 3 and 14.

Next phase of energization is associated with the instabilities and the rate of energy gain is relatively small and it lost over $50<t<500$. Soon after the magnetic reconnection begins, the heating rate increases as seen for $t>550$. The increase in the heating rate is caused by the induced electric fields associated with the magnetic reconnection. Over a short span of time electrons are accelerated to energies several hundred times their initial energies. Ions are also accelerated but to a lesser extent.

\section{Anomalous resistivity}

Dupree (1970) postulated a theoretical framework for anomalous resistivity in plasmas driven by applied electric fields. It involves formation of electron and ion clumps interacting as macro-particles via the fields generated by them. We already saw the clumping in Fig. 8. The interaction gives rise to a drag force on the electrons tantamount to an enhanced plasma resistivity given by

$\eta_{D}=8 \times 10^{-3}\left(k \lambda_{d}\right)\left(\epsilon_{o} \omega_{p e}\right)^{-1} \Omega-m$,

where $k$ is wave number, $\epsilon_{o}$ is the electrical permittivity of free space and the subscript ' $D$ ' refers to Dupree (1970). The foregoing is the Eq. (8) of Dupree (1970), except for a constant converting from CGS to SI units. From Eq. (2) we obtain a theoretical value of the resistivity 


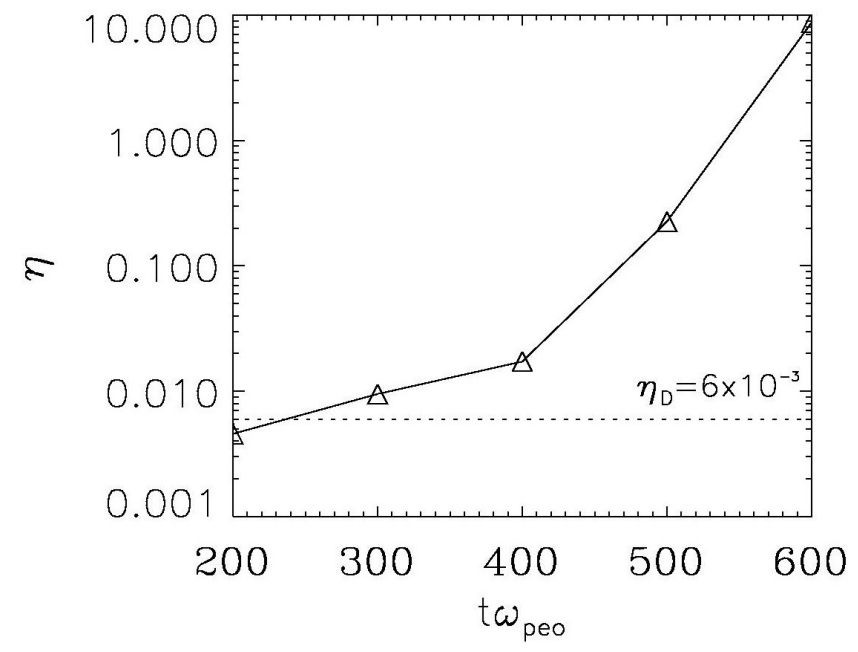

Fig. 16. Anomalous resistivity determined using Eq. (3). The dotted-line curve shows the resistivity level given by the theory of Dupree (1970).

$\eta_{D} \sim 0.01 k \lambda_{d}\left(\omega_{p e} \epsilon_{o}\right)^{-1}$. In the early stage of the instability we have $k \lambda_{d} \sim k_{y} \lambda_{d} \sim 0.6$ and $\eta_{D} \sim 6 \times 10^{-3}\left(\omega_{p e} \epsilon_{o}\right)^{-1}$.

We measure the anomalous resistivity $(\eta)$ in the simulation by the heating rate of the electrons given by

$d W_{e} / d t \sim(1 / 2) \eta<J_{e y}^{2}(t)>/ n_{e}(t)$

Using the plot for $W_{e}(t)$ in Fig. 15, and the average value of the electron current density and number density $n_{e}(t)$ near $z=32$, we have plotted $\eta(t)$ in Fig. 16. For the purpose of comparison, $\eta_{D}$ is also plotted. It is interesting to note that during the early stage of the ion-mode instability, the anomalous resistivity seen from the simulation agrees well with the theoretical value. But at later times the former exceeds the latter by orders of magnitude. We point out that although anomalous resistivity develops in the CS, it does not seem to play a central role in the CS electrodynamics as discussed in this paper. It is the shear driven explosive instability, which seems to play the dominant role. Only in the initial stage of the instability, the anomalous resistivity helps in initiating the onset of shear.

\section{Conclusions and discussion}

Our main purpose of this paper is to study the electrodynamics of a thin current sheet. Our simulations have revealed a rich set of processes including (i) excitation of waves by the current, (ii) partial current disruption by the waves in the central CS setting up sheared flow of the current-carrying electrons, (iii) shear-driven explosive waves, which further disrupt the current, (iv) patchy current disruptions causing magnetic reconnection, ( $v$ ) large heating of both electrons and ions, (vi) nearly complete bifurcation of the original CS and (vii) generation of anomalous resistivity of different levels in the course of the bifurcation of the CS.

We suggest that the initial instability, which occurs in the null magnetic field region and begins to disrupt the current, is likely to lead to the explosive stage by sowing the seed for the initial shear. Lui et al. (1992) have studied a number of such so-called cross-field instabilities in connection with current disruptions in the geotail. These instabilities are modified two-stream, Weibel and electron-acoustic modes with a small but non-zero $B_{z}$ component of the magnetic field in the neutral plane. Further studies are needed to determine the effectiveness of these cross-field instabilities in initiating the shear formation.

For solar flare models it is suggested by Priest (1976) that when $V_{d e}$ exceeds $V_{t e}$, the anomalous resistivity associated with the ion-acoustic modes diffuses the current and reduces the drift velocity and thus it does not induce magnetic connection. We find that diffusion does occur, but the generation of the shear by the local reduction of $V_{d e}$ near the neutral plane makes all the difference in driving the explosive instability and hence magnetic reconnection and faster accelerations of electrons and ions.

Is it possible to have the large anti-parallel magnetic fields with sufficiently small scale lengths to yield a large electron drift to excite the Buneman or ion modes in laboratory and space plasmas? In the laboratory experiment of Baum and Bratenahl (1976) the electron drift reached or exceeded the electron thermal velocity near the so-called flash point. In a series of recent reports on EMHD simulations of magnetotail Ma and Bhattacharjee (1999) have reported sudden impulsive increase in current density $J_{y}$ by two orders of magnitude. Depending on local plasma temperature and density, it is quite likely that Buneman threshold can be met in such events. Exceeding the initial threshold may also critically depend on the composition of the plasma and temperatures of the constituent electrons and ions. Presence of a cold plasma population in the neutral sheet region may satisfy the initial threshold and ignite the explosive instability. Thin current sheets are a consequence of continued forcing in MHD plasma flows as envisioned by Parker (1988) for the solar coronal heating. A systematic study incorporating MHD modeling of large-scale flows and PIC simulations of small-scale microphysics is needed to determine the various types of kinetic plasma processes, depending on the thickness of a current sheet as it evolves from a thick to thin sheet.

It is important to point out that bifurcated current sheets have been observed in the magnetotail by Geotail (Hoshino, et al., 1996) and more recently by Cluster (Nakamura et al., 2003; Segeev et al., 2003). Sitnov et al. (2003) recently proposed a static model depending on pancake type anisotropy of ions. Our simulation shows that the explosive inflectional instability disrupts the current in the central CS and causes the bifurcation of the current sheet. In the region of the disrupted current the magnetic structure becomes complex consisting of evolving loops and reconnecting magnetic field lines. Such magnetic field structures are like those seen in MHD turbulence (Wu and Chang, 2000; Matthaeus et al., 2003).

Finally we comment that Drake et al. (2003) have reported results from 3-D PIC simulations of magnetic reconnection, in which an $x$-line was initialized. Thus initialized reconnection produced fast electron beams, which drove 
Buneman instability resulting in the energization of electrons and ions. We find that initially accelerated electrons in the process of setting up the CS drive the Buneman-like ion mode instabilities, which generate a sheared flow of the current-carrying electrons. The shear-driven instabilities become explosive and drive a variety of processes including clumping of the plasma, magnetic reconnection, bifurcation of the CS, energization of electrons and ions, and generation of anomalous resistivity.

We point out that our simulations have the limitation of small mass ratio $M / m$. In future simulations, this limitation needs to be removed and our findings further verified. However, we note that the Bunneman-like ion mode instability and its consequences are well known in plasma physics. A realistic mass scale is likely to increase the time scale of the evolution. Shear-driven instability is also a well-known one (Ganguli et al., 1988; Mikhailovskii, 1974). The significant contribution in this paper is to report that these instabilities could occur in thin CS and have important consequences for its electrodynamics.

Acknowledgements. Help from S. Saha in some of the early computational work and from N. Puthumbhakum in both computation and visualization are acknowledged. This work was performed under the support of NASA grant NAG5-13489.

Edited by: J. Büchner

Reviewed by: two referees

\section{References}

Baum, P. J. and Bratenahl, A.: Laboratory solar flares experiment, Solar Phys., 47, 331, 1976.

Birdsall, C. K. and Langdon, A. B.: Plasma Physics via Computer Simulations, NY, McGraw-Hill, 1985.

Daughton, W.: The unstable Eigenmodes of neutral sheets, Phys. Plasmas, 6, 1329, 1999.

Drake, J. F., Swisdak, M., Cattell, C., Shay, M. A., Rogers, B. N., and Zeiler, A.: Formation of Electron Holes and Particle Energization During Magnetic Reconnection, Science, 299, 873, 2003

Dupree, T. H.: Theory of resistivity in collisionless plasma, Phys. Rev. Lett., 25, 789, 1970.

Fried, B. D. and Gould, R. W.: Longitudinal ion oscillations in hot plasmas, Phys. Fluids, 4, 139, 1961.

Ganguli, G., Lee, Y. C., and Palmadesso, P. J.: Phys. Fluids, Electron-ion hybrid mode due to transverse shear, Phys. Fluids, 31, 823, 1988.

Harris, E. G.: On a plasma sheath separating regions of oppositely directed magnetic fields, Nuovo Cimento, 23, 1115, 1962.

Hasegawa, A.: Plasma Instabilities and Nonlinear Effects, Berlin, Springer-Verlag, 2, 34, 1975.

Hesse, M. and Birn, J.: Magnetic reconnection: Three-dimensional Aspects and onset in the magnetotail, IEEE Trans. Plasma Sci., 28, 1887, 2000.

Hesse, M., Birn, J., and Kuznetsova, M.: Collisionless magnetic reconnection: Electron processes and transport modeling, J. Geophys. Res., 106, 3721, 2001.

Hoshino, M., Nishida, A., Mukai, T., Saito, Y., and Yamamoto, T.: Structure of plasma sheet in magnetotail: double-peaked electric current sheet, J. Geophys. Res., 101, 24 775, 1996.
Kropotkin, A. P., Trubachev, O. O., and Lui, A. T. Y.: Nonlinear instability of the geomagnetotail current sheet combining the features of tearing and cross-field instabilities, J. Geophys. Res., 104, 371, 1999.

Langdon, A. B.: On enforcing Gauss' law in electromagnetic particle-in-cell codes, Compt. Phys. Commun., 70, 447, 1992.

Lui, A. T. Y.: Tutorial on geomagnetic storms and substorms, IEEE Trans. Plasma Sci., 28, 1854, 2000.

Ma, Z. W. and Bhattachargee, A: Sudden disruption of a thin current sheet in collisionless Hall magnetohydrodynamics due to secondary tearing and coalescence instability, Geophys. Res. Lett., 26, 3337, 1999.

Matthaeus, W. H., Mullan, D. J., Dmitruk, P., et al.: MHD turbulence and heating of the corona and solar wind, Nonl. Proc. Geophys. 10, 93, 2003.

Mikhailovskii, A. B.: Theory of Plasma Instabilities, Consultants Bureau, New York, 7, 141, 1974.

Nakamura, R., Baumjohann, W. Runov, A., et al.: Fast flow during sheet thinning, Geophys. Res. Lett., 29(23), 2140, doi:10.1029/2002GL016200, 2002.

Ozaki, M., Sato, T., Horiuchi, R., and Group, C. S.: Electromagnetic instability and anomalous instability in a neutral sheet, Phys. Plasmas, 3, 2265, 1996.

Parker, E. N.: Nanoflares and the solar x-ray corona, Ap. J., 330, 474, 1988.

Priest, E. R.: Current sheet models of solar flares, Solar Phys., 47, 41, 1976.

Pritchett, P. L.: Geospace environment modeling magnetic reconnection challenge: Simulation with a full particle electromagnetic code, J. Gephys. Res., 106, 3783, 2001.

Pritchett, P. L.: Particle-in-Cell simulations of magnetosphere electrodynamics, IEEE Trans. Plasma Sci., 28, 1976, 2000.

Pritchett, P. L., Coroniti, F. V., and Decyk, V. K.: Three-dimensional stability of thin quasi-neutral current sheets, J. Geophys. Res., 101, 27 413, 1996.

Romero, H. and Ganguli, G.: Nonlinear evolution of a strongly sheared cross-field plasma flow, Phys. Fluids B, 5, 3163, 1993.

Saha, S. and Singh, N.: "Evolution of a plasma to the Harris Equilibrium," paper \#SM21B-11, EOS Trans. AGU, Fall Meeting Suppl. Abstract SM21B-11, 1999.

Segeev, V., Runov, A, Baumjohann, W., et al.: Current sheet flapping motion and structure observed by Cluster, Geophys. Res Lett., 30(6), 1327, 2003.

Shay, M. A., Drake, J. F., Rogers, B. N., Denton, R. E.: Alfvénic collisionless magnetic reconnection and the Hall term, J. Gephys. Res., 106, 3759, 2001.

Silin, I. and Büchner, J.: Kinetic Instabilities of thin current sheets: Results of two-and- one-half-dimensional Vlasov code simulations, Phys. Plasmas, 10, 1299, 2003a.

Silin, I. and Büchner, J.: Nonlinear instability of thin current sheets in antiparallel and guided magnetic fields, Phys. Plasmas, 10, 3561, 2003b.

Sitnov, M. I., Gujdar, P. N., and Swisdak, M.: A model of the bifurcated current sheet, Geophys. Res. Lett., 30, 1721, 2003.

Zeiler, A., Biskamp, D., Drake, J. F., Rogers, B. N., and Shay, M. A. J.: Three dimensional particle simulations of collisionless magnetic reconnection, Geophys. Res., 107, 1230, 2002.

Wu, C. C. and Chang, T.: Dynamical evolution of coherent structures in intermittent two-dimensional MHD turbulence, IEEE Trans. 28, 1938, 2000. 